\title{
SCHUR COMPLEMENTS OF MATRICES WITH ACYCLIC BIPARTITE GRAPHS*
}

\author{
T. BRITZ ${ }^{\dagger}$, D. D. OLESKY ${ }^{\ddagger}$, AND P. VAN DEN DRIESSCHE $^{\dagger}$
}

\begin{abstract}
Bipartite graphs are used to describe the generalized Schur complements of real matrices having no square submatrix with two or more nonzero diagonals. For any matrix $A$ with this property, including any nearly reducible matrix, the sign pattern of each generalized Schur complement is shown to be determined uniquely by the sign pattern of $A$. Moreover, if $A$ has a normalized $L U$ factorization $A=L U$, then the sign pattern of $A$ is shown to determine uniquely the sign patterns of $L$ and $U$, and (with the standard LU factorization) of $L^{-1}$ and, if $A$ is nonsingular, of $U^{-1}$. However, if $A$ is singular, then the sign pattern of the Moore-Penrose inverse $U^{\dagger}$ may not be uniquely determined by the sign pattern of $A$. Analogous results are shown to hold for zero patterns.
\end{abstract}

Key words. Schur complement, LU factorization, Bipartite graph, Sign pattern, Zero pattern, Nearly reducible matrix, Minimally strongly connected digraph.

AMS subject classifications. 05C50, 15A09, 15A23.

1. Introduction. Let $\mathcal{A}$ denote the class of real matrices with acyclic bipartite graphs. In [2], each matrix $A \in \mathcal{A}$ is shown to have a signed generalized inverse, i.e., the sign pattern of the Moore-Penrose inverse $A^{\dagger}$ is determined uniquely by the sign pattern of $A$. If $W$ is a nonsingular square submatrix of a square matrix $A$, then the (classical) Schur complement of $W$ in $A$ is a well-known and useful tool in matrix theory and applications (see, e.g., [10]) that arises in Gaussian elimination. By using the Moore-Penrose inverse, the generalized Schur complement of $W$ in $A$ can be defined for any (singular or nonsquare) submatrix $W$ of $A$ [4], and is denoted by $A / W$.

Our aim here is to use the results of [2] to determine the entries of $A / W$ in terms of those of $A$ for $A \in \mathcal{A}$. In the spirit of [7] for classical Schur complements and [2, 8], we give qualitative results about the sign pattern and zero pattern of $A / W$. For a matrix $A \in \mathcal{A}$ having a normalized $L U$ factorization $A=L U$ (and for a square matrix $A \in \mathcal{A}$ having a standard $L U$ factorization), we also consider qualitative results on the matrices $L, U, L^{-1}$, and, if $A$ is nonsingular, the matrix $U^{-1}$.

Since from [2] each nearly reducible matrix $A$ is a member of $\mathcal{A}$, our results give information about this interesting class of matrices. In particular, the sign (resp., zero) pattern of each generalized Schur complement of a nearly reducible matrix $A$ is determined uniquely by the sign (resp., zero) pattern of $A$. Furthermore, if a nearly reducible matrix $A$ has a normalized $L U$ factorization $A=L U$, the sign (resp., zero)

${ }^{*}$ Received by the editors 7 July 2004. Accepted for publication 29 December 2004. Handling Editor: Bryan L. Shader.

${ }^{\dagger}$ Department of Mathematics and Statistics, University of Victoria, Victoria, BC V8W 3P4, Canada (pvdd@math.uvic.ca). This work was supported by an NSERC Discovery Grant. T. Britz was also supported by a PIMS Postdoctoral Fellowship. Current address for T. Britz is Department of Mathematics, Technical University of Denmark, Bygning 303, 2800 Kgs. Lyngby, Denmark (T.Britz@mat.dtu.dk).

${ }^{\ddagger}$ Department of Computer Science, University of Victoria, Victoria, BC V8W 3P6, Canada (dolesky@cs.uvic.ca). This work was supported by an NSERC Discovery Grant. 
pattern of $A$ determines uniquely the sign (resp., zero) patterns of $L, U, L^{-1}$, and also, if $A$ is nonsingular, the sign (resp., zero) pattern of $U^{-1}$.

2. Generalized Schur complements. For any real $m \times n$ matrix $A=\left[a_{i j}\right]$, the Moore-Penrose inverse $A^{\dagger}$ is the unique matrix that satisfies the following four properties $[9,11]$ :

$$
A^{\dagger} A A^{\dagger}=A^{\dagger} \quad A A^{\dagger} A=A \quad\left(A^{\dagger} A\right)^{T}=A^{\dagger} A \quad\left(A A^{\dagger}\right)^{T}=A A^{\dagger} .
$$

If $A$ is a square, nonsingular matrix, then $A^{\dagger}=A^{-1}$. Thus, Moore-Penrose inversion generalizes standard matrix inversion. Let $B(A)$ be the bipartite graph with vertices $\left\{u_{1}, \ldots, u_{m}, v_{1}, \ldots, v_{n}\right\}$ and edges $\left\{\left\{u_{i}, v_{j}\right\} \mid a_{i j} \neq 0\right\}$. Let $\mathcal{B}$ denote the family of finite acyclic bipartite graphs, and let $\mathcal{A}$ denote the family of all real matrices $A$ with $B(A) \in \mathcal{B}$. If $A$ is an $n \times n$ matrix, then a nonzero diagonal of $A$ is a collection of $n$ nonzero entries of $A$, no two of which lie in the same row or in the same column. Note that $\mathcal{A}$ consists of all real matrices that contain no square submatrix with more than one nonzero diagonal. A matching in a (bipartite) graph is a subset of its edges no two of which are adjacent. For $t \geq 0$ and any bipartite graph $B$, let $M_{t}(B)$ denote the family of matchings in $B$ that contain $t$ edges.

Theorem 2.1. [2] Let $A=\left[a_{i j}\right] \in \mathcal{A}$ be an $m \times n$ matrix with rank $r \geq 2$, and let $A^{\dagger}=\left[\alpha_{i j}\right]$ denote the Moore-Penrose inverse of $A$. If $B(A)$ contains a path $p$ from $u_{i}$ to $v_{j}$

$$
u_{i} \rightarrow v_{j_{1}} \rightarrow u_{i_{1}} \rightarrow v_{j_{2}} \rightarrow u_{i_{2}} \rightarrow \cdots \rightarrow v_{j_{s}} \rightarrow u_{i_{s}} \rightarrow v_{j}
$$

of length $2 s+1$ with $s \geq 0$, then

$$
\alpha_{j i}=(-1)^{s} a_{i j_{1}} a_{i_{1} j_{1}} a_{i_{1} j_{2}} \cdots a_{i_{s} j_{s}} a_{i_{s} j} \frac{\sum_{\substack{E \in M_{r-s-1}(B(A)) \\ V(E) \cap V(p)=\emptyset}} \prod_{\left\{u_{k}, v_{l}\right\} \in E}\left(a_{k l}\right)^{2}}{\sum_{F \in M_{r}(B(A))} \prod_{\left\{u_{k}, v_{l}\right\} \in F}\left(a_{k l}\right)^{2}} .
$$

Otherwise, $\alpha_{j i}=0$.

Note that when $s=0$, the product $a_{i j_{1}} a_{i_{1} j_{1}} a_{i_{1} j_{2}} \cdots a_{i_{s} j_{s}} a_{i_{s} j}$ reduces to $a_{i j}$, and that when $r-s-1=0$, the numerator in the quotient of summations is equal to 1 .

Let

$$
A=\left[\begin{array}{cc}
W & X \\
Y & Z
\end{array}\right]
$$

be an $m \times n$ matrix, where $W$ is a $k \times l$ matrix with $k \leq m-1, l \leq n-1$, and rank $r$. Let $A / W$ denote the (generalized) Schur complement of $W$ in $A$ (see [4]),

$$
A / W=Z-Y W^{\dagger} X
$$

Let the rows and columns of $A / W$ be indexed by the indices $i=k+1, \ldots, m$ and $j=l+1, \ldots, n$, respectively, and let the rows and columns of $W=\left[w_{p q}\right], X=\left[x_{p j}\right]$, $Y=\left[y_{i q}\right]$, and $Z=\left[z_{i j}\right]$ be indexed as in $A(p=1, \ldots, k ; q=1, \ldots, l)$. 
TheOREm 2.2. Suppose that $A=\left[a_{i j}\right] \in \mathcal{A}$ is an $m \times n$ matrix partitioned as in (2.1) and rank $W=r \geq 2$. Let $i, j$ be integers such that $k+1 \leq i \leq m$ and $l+1 \leq j \leq n$. If $B(Y)$ and $B(X)$ contain edges $\left\{u_{i}, v_{i^{\prime}}\right\}$ and $\left\{u_{j^{\prime}}, v_{j}\right\}$, respectively, and $B(W)$ contains a path $p$ from $v_{i^{\prime}}$ to $u_{j^{\prime}}$

$$
v_{i^{\prime}} \rightarrow u_{j_{1}} \rightarrow v_{i_{1}} \rightarrow u_{j_{2}} \rightarrow v_{i_{2}} \rightarrow \cdots \rightarrow u_{j_{s}} \rightarrow v_{i_{s}} \rightarrow u_{j^{\prime}}
$$

of length $2 s+1$ with $s \geq 0$, then the entry $(A / W)_{i j}$ equals

$$
(-1)^{s+1} a_{i i^{\prime}} a_{j^{\prime} i_{s}} a_{j_{s} i_{s}} \cdots a_{j_{2} i_{1}} a_{j_{1} i_{1}} a_{j_{1} i^{\prime}} a_{j^{\prime} j} \frac{\sum_{\substack{E \in M_{r-s-1}(B(W)) \\ V(E) \cap V(p)=\emptyset}} \prod_{\left\{u_{k}, v_{l}\right\} \in E}\left(a_{k l}\right)^{2}}{\sum_{F \in M_{r}(B(W))} \prod_{\left\{u_{k}, v_{l}\right\} \in F}\left(a_{k l}\right)^{2}} .
$$

Otherwise, $(A / W)_{i j}=a_{i j}=z_{i j}$.

Proof. By definition,

$$
(A / W)_{i j}=z_{i j}-\sum_{i^{\prime}, j^{\prime}} y_{i i^{\prime}}\left(W^{\dagger}\right)_{i^{\prime} j^{\prime}} x_{j^{\prime} j}
$$

If the edges and the path exist as given in the theorem, then

$$
u_{i} \rightarrow v_{i^{\prime}} \rightarrow u_{j_{1}} \rightarrow v_{i_{1}} \rightarrow u_{j_{2}} \rightarrow v_{i_{2}} \rightarrow \cdots \rightarrow u_{j_{s}} \rightarrow v_{i_{s}} \rightarrow u_{j^{\prime}} \rightarrow v_{j}
$$

is a path in $B(A)$ from $u_{i}$ to $v_{j}$. Since $B(A)$ is acyclic, $z_{i j}=0$ and there are no other paths from $u_{i}$ to $v_{j}$. Thus, the sum above consists of the single term $y_{i i^{\prime}}\left(W^{\dagger}\right)_{i^{\prime} j^{\prime}} x_{j^{\prime} j}$. The first part of the theorem now follows from Theorem 2.1. If, however, such edges and path do not exist, then by Theorem 2.1, $y_{i i^{\prime}}\left(W^{\dagger}\right)_{i^{\prime} j^{\prime}} x_{j^{\prime} j}=0$ for all $i^{\prime}, j^{\prime}$. Hence, $(A / W)_{i j}=a_{i j}=z_{i j}$.

For completeness, results analogous to Theorem 2.2 are now stated for the cases rank $W \leq 1$.

REMARK 2.3. Suppose that $A \in \mathcal{A}$ is partitioned as in (2.1). If rank $W=0$, then clearly $A / W=Z$. Suppose that $\operatorname{rank} W=1$ and that $i, j$ are as in Theorem 2.2. If $B(Y), B(W)$, and $B(X)$ contain edges $\left\{u_{i}, v_{i^{\prime}}\right\},\left\{v_{i^{\prime}}, u_{j^{\prime}}\right\}$, and $\left\{u_{j^{\prime}}, v_{j}\right\}$, respectively, then

$$
(A / W)_{i j}=-\frac{y_{i i^{\prime}} w_{j^{\prime} i^{\prime}} x_{j^{\prime} j}}{\sum_{t=1}^{k} \sum_{q=1}^{l} w_{t q}^{2}}
$$

Otherwise, $(A / W)_{i j}=a_{i j}=z_{i j}$.

COROLlary 2.4. $B(A / W)$ contains an edge $\left\{u_{i}, v_{j}\right\}$ if and only if one of the following two mutually exclusive statements is true:

1. $B(A)$ contains a path $p$ from $u_{i}$ to $v_{j}$ of length $2 s+1$ with $s \geq 0$ with all intermediate vertices in $B(W)$, and $B(W) \backslash V(p)$ contains a matching with $r-s-1$ edges; 
2. $B(A)$ contains the edge $\left\{u_{i}, v_{j}\right\}$.

The sign pattern of any real matrix is the matrix obtained by replacing each negative entry in the matrix by a minus sign $(-)$, and each positive entry in the matrix by a plus sign $(+)$. The zero pattern of any real matrix is the matrix obtained by replacing each nonzero entry in the matrix by an asterisk $(*)$. By Theorem 2.1, the sign (resp., zero) pattern of a matrix $A \in \mathcal{A}$ determines uniquely the sign (resp., zero) pattern of $A^{\dagger}$.

Corollary 2.5. For any $A \in \mathcal{A}$, the sign pattern of each Schur complement of $A$ is determined uniquely by the sign pattern of $A$. Furthermore, the zero pattern of each Schur complement of $A$ is determined uniquely by the zero pattern of $A$.

Proof. For any submatrix $W$ of $A$, let $P$ and $Q$ be permutation matrices such that $W$ is a leading submatrix of $P A Q$. Since $P A Q \in \mathcal{A}$, the result follows from Theorem 2.2. [

EXAMPLE 2.6. Let

$$
A=\left[\begin{array}{ccc|cc}
a_{11} & a_{12} & 0 & 0 & 0 \\
0 & a_{22} & 0 & a_{24} & 0 \\
\hline a_{31} & 0 & 0 & 0 & a_{35}
\end{array}\right]=\left[\begin{array}{cc}
W & X \\
Y & Z
\end{array}\right] \in \mathcal{A},
$$

where each entry $a_{i j}$ is nonzero. The associated bipartite graphs are displayed in Figure 2.1. Note that $B(Y)$ and $B(X)$ contain the edges $\left\{u_{3}, v_{1}\right\}$ and $\left\{u_{2}, v_{4}\right\}$, respectively, and that $B(W)$ contains the path $v_{1} \rightarrow u_{1} \rightarrow v_{2} \rightarrow u_{2}$. By Theorem 2.2,

$$
(A / W)_{34}=(-1)^{2} \frac{a_{31} a_{22} a_{12} a_{11} a_{24}}{a_{11}^{2} a_{22}^{2}}=\frac{a_{31} a_{12} a_{24}}{a_{11} a_{22}} .
$$

(Here $r=2,(i, j)=(3,4), s=i^{\prime}=j_{1}=1, j^{\prime}=i_{1}=2$, and $r-s-1=0$.) However, since $B(X)$ has no edge that is adjacent to the vertex $v_{5}$, it follows from Theorem 2.2 that $(A / W)_{35}=a_{35}=z_{35}$.

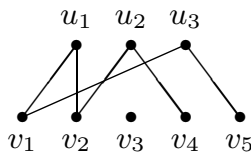

$B(A)$

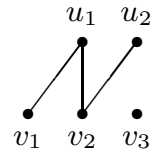

$B(W)$

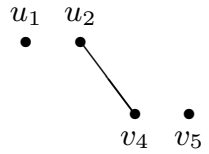

$B(X)$

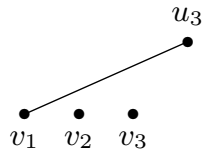

$B(Y)$

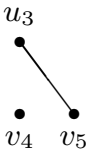

$B(Z)$

FIG. 2.1. Bipartite graphs for Example 2.6

3. Sign and zero patterns and $L U$ factorization. Let $A$ be an $m \times n$ matrix. For any strictly increasing sequences of integers $\gamma \in(1, \ldots, m)$ and $\delta \in(1, \ldots, n)$, let $A[\gamma \mid \delta]$ denote the submatrix of $A$ whose rows and columns are indexed by $\gamma$ and $\delta$, respectively. For $k=1, \ldots, n$, let $A[k]$ denote the matrix $A[1, \ldots, k \mid 1, \ldots, k]$. An $m \times r$ matrix $L=\left[l_{i j}\right]$ with $r \leq m$ is lower trapezoidal if $l_{i j}=0$ for all $i<j$; similarly, an $r \times n$ matrix $U=\left[u_{i j}\right]$ with $r \leq n$ is upper trapezoidal if $u_{i j}=0$ for all $i>j$. If $m=r$, then $L$ is lower triangular; similarly if $r=n$, then $U$ is upper triangular. An 
$m \times n$ matrix $A$ with rank $r \geq 1$ has an $L U$ factorization if there exist an $m \times r$ lower trapezoidal matrix $L$ and an $r \times n$ upper trapezoidal matrix $U$ such that $A=L U[12$, Section 2.6]. If $l_{i i}=1$ for each $i=1, \ldots, r$, then the $L U$ factorization is unique and is said to be normalized. It is shown below that if $A \in \mathcal{A}$, then Theorem 2.2 can be applied to determine the entries of $L$ and $U$.

By the results in $[5$, p. 27] when $A$ is square, $A$ has a normalized $L U$ factorization if and only if $\operatorname{det} A[k] \neq 0$ for each $k=1, \ldots, r$. Furthermore by [5, p. 26], if $\operatorname{det} A[k] \neq 0$ for $k=1, \ldots, r$, then for all $i=k+1, \ldots, m$ and $j=k+1, \ldots, n$,

$$
(A / A[k])_{i j}=\frac{\operatorname{det} A[1, \ldots, k, i \mid 1, \ldots, k, j]}{\operatorname{det} A[k]} .
$$

The first row of $U$ is equal to the first row of $A$, and the first column of $L$ is equal to the first column of $A$ multiplied by the scalar $1 / a_{11}$. For any $i=2, \ldots, r$ and $j=i, \ldots, n$,

$$
u_{i j}=\frac{\operatorname{det} A[1, \ldots, i \mid 1, \ldots, i-1, j]}{\operatorname{det} A[i-1]}=(A / A[i-1])_{i j}
$$

and for any $i=2, \ldots, r$ and $j=i, \ldots, m$,

$$
l_{j i}=\frac{\operatorname{det} A[1, \ldots, i-1, j \mid 1, \ldots, i]}{\operatorname{det} A[i]}=\frac{(A / A[i-1])_{j i}}{(A / A[i-1])_{i i}} .
$$

For the above details when $A$ is square, see [5, p. 35-36]. If $A \in \mathcal{A}$, then the entries $u_{i j}$ and $l_{j i}$ can be easily found from (3.2) and (3.3) either by evaluating the determinants or by using Theorem 2.2 to evaluate the appropriate entries of the Schur complement $A / A[i-1]$.

Theorem 3.1. Let $A \in \mathcal{A}$ be an $m \times n$ matrix with rank $r \geq 1$, and let $P, Q$ be permutation matrices such that $P A Q$ has a normalized $L U$ factorization $P A Q=L U$. The sign patterns of $L$ and $U$ are determined uniquely by the sign pattern of $A$. Furthermore, the zero patterns of $L$ and $U$ are determined uniquely by the zero pattern of $A$.

Proof. By (3.2), (3.3), and the sentence before (3.2), the sign patterns of $U$ and $L$ are determined uniquely by the signs of certain minors of $P A Q$. Since $P A Q \in \mathcal{A}$, the signs of these minors are determined uniquely by the sign pattern of $P A Q$, and thus by the sign pattern of $A$. Similarly, the zero patterns of $U$ and $L$ are determined uniquely by whether or not certain minors of $P A Q$ equal zero, and thus by the zero pattern of $A$. Note that Theorem 3.1 also follows from Corollary 2.5.

In the terminology of [8], Theorem 3.1 states that for $A \in \mathcal{A}$ and the normalized $L U$ factorization $P A Q=L U$, the entries of $L$ and $U$ are unambiguous; that is, for every real matrix $B$ with the same sign pattern as $A$, if $P B Q=\widehat{L} \widehat{U}$ is the normalized $L U$ factorization, then the sign patterns of $L$ and $\widehat{L}$ are the same, as are the sign patterns of $U$ and $\widehat{U}$.

To prove Theorems 3.3 and 3.4 below, the following lemma is required.

Lemma 3.2. Let $A=\left[a_{i j}\right]$ be any nonsingular $n \times n$ matrix with $n \geq 2$ and $a$ normalized $L U$ factorization $A=L U$, and let $L^{-1}=\left[\lambda_{i j}\right]$ and $U^{-1}=\left[\mu_{i j}\right]$. Then 
$\lambda_{11}=1, \mu_{11}=\frac{1}{a_{11}}$, and for integers $i=2, \ldots, n$ and $j=1, \ldots, i$,

$$
\lambda_{i j}=(-1)^{i+j} \frac{\operatorname{det} A[(1, \ldots, i)-j \mid 1, \ldots, i-1]}{\operatorname{det} A[i-1]},
$$

and

$$
\mu_{j i}=(-1)^{i+j} \frac{\operatorname{det} A[1, \ldots, i-1 \mid(1, \ldots, i)-j]}{\operatorname{det} A[i]}
$$

Proof. Clearly, $L^{-1}$ is lower triangular with $\lambda_{11}=\cdots=\lambda_{n n}=1$, so the above expression for $\lambda_{i i}$ is correct. Also, $U^{-1}$ is upper triangular with $\mu_{i i}=\frac{1}{u_{i i}}$, so by (3.2), the above expression for $\mu_{i i}$ is correct. Suppose now that $i, j$ are integers such that $1 \leq j<i \leq n$. Let $R=\left[\delta_{i, n+1-i}\right]$ denote the reverse diagonal permutation matrix, i.e., the permutation matrix that corresponds to the involution $(1, \ldots, n) \mapsto(n, \ldots, 1)$, and let

$$
A^{\prime}=R\left(A^{-1}\right)^{T} R, \quad L^{\prime}=R\left(L^{-1}\right)^{T} R, \quad \text { and } \quad U^{\prime}=R\left(U^{-1}\right)^{T} R .
$$

Then $A^{\prime}=L^{\prime} U^{\prime}$ is the normalized $L U$ factorization of $A^{\prime}$. By (3.3),

$$
\begin{aligned}
\lambda_{i j} & =\left(L^{\prime}\right)_{n+1-j, n+1-i} \\
& =\frac{\operatorname{det} A^{\prime}[1, \ldots, n-i, n+1-j \mid 1, \ldots, n+1-i]}{\operatorname{det} A^{\prime}[n+1-i]} \\
& =\frac{\operatorname{det} A^{-1}[i, \ldots, n \mid j, i+1, \ldots, n]}{\operatorname{det} A^{-1}[i, \ldots, n \mid i, \ldots, n]} .
\end{aligned}
$$

By Jacobi's Theorem (see, e.g., [6, (0.8.4)]),

$$
\lambda_{i j}=(-1)^{i+j} \frac{\operatorname{det} A[(1, \ldots, i)-j \mid 1, \ldots, i-1]}{\operatorname{det} A[i-1]} .
$$

(An analogous formula is given in [8, proof of Theorem 3.3] when $U$ is normalized to have all diagonal entries equal to 1.$)$

By the above method, it may be shown that

$$
\mu_{j i}=\frac{\operatorname{det} A^{-1}[j, i+1, \ldots, n \mid i, \ldots, n]}{\operatorname{det} A^{-1}[i+1, \ldots, n \mid i+1, \ldots, n]}=(-1)^{i+j} \frac{\operatorname{det} A[1, \ldots, i-1 \mid(1, \ldots, i)-j]}{\operatorname{det} A[i]}
$$

which concludes the proof. $\square$

For the case in which $A$ is an $n \times n$ singular matrix with rank $r \geq 1$ and the normalized $L U$ factorization $A=L U$, the matrices $L$ and $U$ can be extended to be lower and upper triangular, respectively. The standard $L U$ factorization of an $n \times n$ singular matrix $A$ with rank $r$ extends the normalized $L U$ factorization so that both $L$ and $U$ are $n \times n$ matrices, $L[1, \ldots, r \mid r+1, \ldots, n]=0, L[r+1, \ldots, n \mid r+1, \ldots, n]=I$, and $U[r+1, \ldots, n \mid 1, \ldots, n]=0$. If $A$ is a nonsingular matrix, then the normalized and standard $L U$ factorizations are the same. 
TheOREM 3.3. If $A \in \mathcal{A}$ is an $n \times n$ matrix with a standard $L U$ factorization $A=L U$, then the sign pattern of $L^{-1}$ is determined uniquely by the sign pattern of $A$, and the zero pattern of $L^{-1}$ is determined uniquely by the zero pattern of $A$.

Proof. Note that $\lambda_{11}=\cdots=\lambda_{n n}=1$. Let $i, j$ be integers such that $1 \leq j<i \leq n$, and let $r=\operatorname{rank} A \geq 1$. If $r=n$, i.e., $A$ is nonsingular, then by Lemma 3.2,

$$
\lambda_{i j}=(-1)^{i+j} \frac{\operatorname{det} A[(1, \ldots, i)-j \mid 1, \ldots, i-1]}{\operatorname{det} A[i-1]} .
$$

Since $A$ is a member of $\mathcal{A}$ with a normalized $L U$ factorization, the submatrix $A[i-1]$ contains precisely one nonzero diagonal, and $A[(1, \ldots, i)-j \mid 1, \ldots, i-1]$ contains at most one nonzero diagonal. Hence, the sign pattern of $A$ determines the sign of $\lambda_{i j}$, and the zero pattern of $A$ determines whether or not $\lambda_{i j}=0$.

Suppose that $1 \leq r \leq n-1$. Since $A$ is a member of $\mathcal{A}$ with a normalized $L U$ factorization, $A$ may be written as

$$
A=\left[\begin{array}{cc}
A_{1} & A_{2} \\
A_{3} & 0
\end{array}\right]
$$

where $A_{1}=A[r]$ is nonsingular and has precisely one nonzero diagonal. Note that

$$
L=\left[\begin{array}{ll}
L_{1} & 0 \\
L_{2} & I
\end{array}\right] \quad \text { and } \quad U=\left[\begin{array}{cc}
U_{1} & U_{2} \\
0 & 0
\end{array}\right]
$$

where $L_{1}=L[r]$ and $U_{1}=U[r]$ are nonsingular. Define

$$
A^{\prime}=\left[a_{i j}^{\prime}\right]=\left[\begin{array}{cc}
A_{1} & A_{2} \\
A_{3} & I
\end{array}\right], \quad \text { and } \quad U^{\prime}=\left[\begin{array}{cc}
U_{1} & U_{2} \\
0 & I
\end{array}\right] .
$$

Then $A^{\prime}$ is nonsingular with normalized $L U$ factorization $A^{\prime}=L U^{\prime}$. By Lemma 3.2,

$$
\lambda_{i j}=(-1)^{i+j} \frac{\operatorname{det} A^{\prime}[(1, \ldots, i)-j \mid 1, \ldots, i-1]}{\operatorname{det} A^{\prime}[i-1]} .
$$

To prove the theorem, it suffices to show that $A^{\prime}[i-1]$ has precisely one nonzero diagonal, and that $A^{\prime}[(1, \ldots, i)-j \mid 1, \ldots, i-1]$ has at most one nonzero diagonal. It may be assumed without loss of generality that the nonzero diagonal of $A^{\prime}[r]=A[r]$ is the main diagonal $\left\{a_{11}, \ldots, a_{r r}\right\}$.

If $i \leq r+1$, then $A^{\prime}[i-1]=A[i-1] \in \mathcal{A}$, so suppose that $i \geq r+2$. The submatrix $A^{\prime}[i-1]$ has the nonzero diagonal

$$
\widehat{D}=\left\{a_{11}^{\prime}, \ldots, a_{r r}^{\prime}, a_{r+1, r+1}^{\prime}, \ldots, a_{i-1, i-1}^{\prime}\right\}
$$

where $a_{k k}^{\prime}=a_{k k}$ for $k=1, \ldots, r$, and $a_{k k}^{\prime}=1$ for $k=r+1, \ldots, i-1$. Suppose that it also contains another diagonal, say $D^{\prime}$. Since $A^{\prime}[r]=A[r] \in \mathcal{A}$ and $\widehat{D} \neq D^{\prime}$, it follows that $D^{\prime}$ cannot contain all of the $i-r-1$ entries $a_{r+1, r+1}^{\prime}, \ldots, a_{i-1, i-1}^{\prime}$. Denote by $D$ the collection of entries obtained by deleting from $D^{\prime}$ all of the entries 
$a_{r+1, r+1}^{\prime}, \ldots, a_{i-1, i-1}^{\prime}$ that are contained in $D^{\prime}$. Then $D$ is a diagonal of some submatrix of $A$ of order at least $r+1$. Since $A \in \mathcal{A}$, the rank of this submatrix, and thus of $A$, is at least $r+1$, a contradiction. Thus, $A^{\prime}[i-1]$ has precisely one nonzero diagonal.

To show that $A^{\prime}[(1, \ldots, i)-j \mid 1, \ldots, i-1]$ has at most one nonzero diagonal, note that if $i \leq r$, then this matrix is a submatrix of $A \in \mathcal{A}$. Suppose then that $i \geq r+1$. Since $i \neq j$, each nonzero diagonal $D^{\prime}$ of $A^{\prime}[(1, \ldots, i)-j \mid 1, \ldots, i-1]$ is of the form

$$
\left\{a_{i_{k} i_{k+1}}^{\prime} \mid k=0, \ldots, t-1\right\} \cup E
$$

where $t \geq 1$ and $i=i_{0}, i_{1}, \ldots, i_{t}=j$ are distinct, and where $E$ is a diagonal of $A^{\prime}\left[(1, \ldots, i-1)-\left\{i_{1}, \ldots, i_{t}\right\}\right]$. (Note that $t \leq i-1$ and that if $t=i-1$, then $E$ is vacuous.) Since $A^{\prime}[i-1]$ has been shown above to contain precisely one nonzero diagonal, namely the nonzero diagonal $\left\{a_{11}^{\prime}, \ldots, a_{i-1, i-1}^{\prime}\right\}$, and $\left\{a_{i_{1} i_{1}}^{\prime}, \ldots, a_{i_{t} i_{t}}^{\prime}\right\} \cup E$ is a nonzero diagonal of $A^{\prime}[i-1]$, these two nonzero diagonals must be identical. Hence,

$$
D^{\prime}=\left\{a_{i_{k} i_{k+1}}^{\prime} \mid k=0, \ldots, t-1\right\} \cup\left\{a_{k k}^{\prime} \mid 1 \leq k \leq i-1, k \neq i_{1}, \ldots, i_{t}\right\} .
$$

Assume that at least one of the indices $i_{1}, \ldots, i_{t}$ is greater than or equal to $r+1$. Let $F=\left\{a_{k k}^{\prime} \in D^{\prime} \mid r+1 \leq k \leq i-1\right\}$. Since for $s \geq 1$ some index $i_{s}$ is greater than or equal to $r+1$, it follows that $0 \leq|F| \leq i-r-2$. Thus, if $D=D^{\prime}-F$, then $|D| \geq r+1$; i.e., $D$ is a diagonal of some submatrix of $A$ of order at least $r+1$. This contradicts the fact that $\operatorname{rank} A=r$; hence, $i_{1}, \ldots, i_{t} \leq r$. For each nonzero diagonal $D^{\prime}$ of $A^{\prime}[(1, \ldots, i)-j \mid 1, \ldots, i-1]$ as above, the bipartite graph $B\left(A^{\prime}\right)$ contains the path

$$
u_{i}=u_{i_{0}} \rightarrow v_{i_{1}} \rightarrow u_{i_{1}} \rightarrow v_{i_{2}} \rightarrow u_{i_{2}} \rightarrow \cdots \rightarrow v_{i_{t}}=v_{j}
$$

Since $i_{1}, \ldots, i_{t} \leq r$, this path is also a path of $B(A)$, from $u_{i}$ to $v_{j}$. Thus if $A^{\prime}[(1, \ldots, i)-j \mid 1, \ldots, i-1]$ has two distinct nonzero diagonals, then $B(A)$ contains two distinct paths from $u_{i}$ to $v_{j}$, and must therefore contain a cycle, which contradicts the acyclicity of $B(A)$. Hence, $A^{\prime}[(1, \ldots, i)-j \mid 1, \ldots, i-1]$ has at most one nonzero diagonal. $\square$

THEOREM 3.4. If $A \in \mathcal{A}$ is nonsingular with normalized $L U$ factorization $A=$ $L U$, then the sign pattern of $U^{-1}$ is determined uniquely by the sign pattern of $A$, and the zero pattern of $U^{-1}$ is determined uniquely by the zero pattern of $A$.

Proof. By Lemma 3.2, $\mu_{11}=\frac{1}{a_{11}}$ and, for $i=2, \ldots, n$ and $j=1, \ldots, i$,

$$
\mu_{j i}=(-1)^{i+j} \frac{\operatorname{det} A[1, \ldots, i-1 \mid(1, \ldots, i)-j]}{\operatorname{det} A[i]} .
$$

Since $A$ is a member of $\mathcal{A}$ with a normalized $L U$ factorization, the submatrix $A[i]$ contains precisely one nonzero diagonal, and $A[1, \ldots, i-1 \mid(1, \ldots, i)-j]$ contains at most one nonzero diagonal. Hence, the sign pattern of $A$ determines the sign of $\mu_{j i}$, and the zero pattern of $A$ determines whether or not $\mu_{j i}=0$.

Suppose that $A \in \mathcal{A}$ has the standard $L U$ factorization $A=L U$. In the terminology of [8], Theorem 3.3 states that $L^{-1}$ is unambiguous. If $A$ is nonsingular, then 
Theorem 3.4 states that $U^{-1}$ is also unambiguous. However, if $A$ is singular, then $U$ is singular and (as the following example shows) the sign and zero patterns of $U^{\dagger}$ are not necessarily determined uniquely by the sign and zero patterns of $A$; that is, they may be ambiguous.

EXAMPLE 3.5. Let

$$
A=\left[\begin{array}{cccccc}
a_{11} & a_{12} & 0 & a_{14} & 0 & 0 \\
0 & a_{22} & a_{23} & 0 & a_{25} & 0 \\
0 & 0 & a_{33} & 0 & 0 & a_{36} \\
a_{41} & 0 & 0 & 0 & 0 & 0 \\
0 & 0 & 0 & 0 & 0 & 0 \\
0 & 0 & 0 & 0 & 0 & 0
\end{array}\right]
$$

where each entry $a_{i j}$ is nonzero. Then $A \in \mathcal{A}$ but the normalized (or standard) $L U$ factorization $A=L U$ has the property that the $(5,3)$ entry of $U^{\dagger}$ equals

$$
\frac{a_{23} a_{25}}{a_{33}} \frac{a_{12}^{2} a_{36}^{2}-a_{14}^{2} a_{33}^{2}}{a_{36}^{2}\left(\left(a_{12}^{2}+a_{14}^{2}\right)\left(a_{23}^{2}+a_{25}^{2}\right)+a_{14}^{2} a_{22}^{2}\right)+a_{33}^{2}\left(a_{14}^{2}\left(a_{22}^{2}+a_{25}^{2}\right)+a_{12}^{2} a_{25}^{2}\right)} .
$$

Thus, this entry is equal to 0 if and only if $a_{12}^{2} a_{36}^{2}=a_{14}^{2} a_{33}^{2}$, which does not depend only on the signs of these entries.

4. Nearly reducible matrices. An irreducible matrix is nearly reducible if it is reducible whenever any nonzero entry is set to zero [3, Section 3.3]. For each $n \times n$ matrix $A=\left[a_{i j}\right]$ with $n \geq 2$, let $D(A)$ be the directed graph with vertices $W=$ $\left\{w_{1}, \ldots, w_{n}\right\}$ and $\operatorname{arcs}\left\{\left(w_{i}, w_{j}\right) \in W \times W \mid a_{i j} \neq 0\right\}$. In terms of digraphs, $A$ is nearly reducible if and only if $D(A)$ is minimally strongly connected, i.e., $D(A)$ is strongly connected but the removal of any arc of $D(A)$ causes the digraph to no longer be strongly connected. It is proved in [2] that every nearly reducible matrix is a member of $\mathcal{A}$. Hence, Theorems 4.1 and 4.2 below follow immediately from Corollary 2.5 and from Theorems 3.1 and 3.3, respectively.

TheOREM 4.1. Let $A$ be a nearly reducible $n \times n$ matrix with $n \geq 2$. For any $n \times n$ permutation matrices $P, Q$, the sign pattern of each Schur complement of $P A Q$ is determined uniquely by the sign pattern of $A$. Furthermore, the zero pattern of each Schur complement of $P A Q$ is determined uniquely by the zero pattern of $A$.

THEOREM 4.2. Let $A$ be a nearly reducible matrix, and let $P, Q$ be permutation matrices such that $P A Q$ has a standard $L U$ factorization $P A Q=L U$. Then the sign patterns of $L, U$, and $L^{-1}$ are determined uniquely by the sign pattern of $A$. Furthermore, the zero patterns of $L, U$, and $L^{-1}$ are determined uniquely by the zero pattern of $A$.

We now restrict consideration to nonsingular nearly reducible matrices, which are shown in [1] to be strongly sign-nonsingular; that is, for such a matrix $A$, the sign pattern of $A^{-1}$ is determined uniquely by the sign pattern of $A$. The next result follows immediately from Theorems $3.3,3.4$ and 4.2 .

THEOREM 4.3. Let $A$ be a nonsingular nearly reducible matrix, and let $P, Q$ be permutation matrices such that $P A Q$ has a normalized $L U$ factorization $P A Q=L U$. Then the sign pattern of $A$ determines uniquely the sign patterns of $L, U, L^{-1}$, and 
$U^{-1}$, and the zero pattern of $A$ determines uniquely the zero patterns of $L, U, L^{-1}$, and $U^{-1}$.

ExAmple 4.4. Consider the following normalized $L U$ factorization $P A=L U$,

$$
\left[\begin{array}{llll}
0 & 1 & 0 & 0 \\
1 & 0 & 0 & 0 \\
0 & 0 & 0 & 1 \\
0 & 0 & 1 & 0
\end{array}\right]\left[\begin{array}{cccc}
0 & a_{12} & 0 & 0 \\
a_{21} & 0 & a_{23} & 0 \\
0 & a_{32} & 0 & a_{34} \\
0 & 0 & a_{43} & 0
\end{array}\right]=\left[\begin{array}{cccc}
1 & 0 & 0 & 0 \\
0 & 1 & 0 & 0 \\
0 & 0 & 1 & 0 \\
0 & \frac{a_{32}}{a_{12}} & 0 & 1
\end{array}\right]\left[\begin{array}{cccc}
a_{21} & 0 & a_{23} & 0 \\
0 & a_{12} & 0 & 0 \\
0 & 0 & a_{43} & 0 \\
0 & 0 & 0 & a_{34}
\end{array}\right],
$$

in which $A$ is nonsingular and nearly reducible, and $P$ is a permutation matrix such that $P A$ has only nonzero entries on the main diagonal. Clearly, the sign (resp., zero) patterns of $L$ and $U$ are determined uniquely by the sign (resp., zero) pattern of $A$. Furthermore, Theorem 4.3 asserts that the sign (resp., zero) pattern of $A$ determines uniquely the sign patterns of $L^{-1}$ and $U^{-1}$.

Finally, we remark that for the normalized $L U$ factorization of the matrix $A$ in Example 3.5 (where $A$ is neither nonsingular nor nearly reducible), the bipartite graph $B(U)$ has cycles of length 6 and 8 , and thus $U \notin \mathcal{A}$. However, $L, U \in \mathcal{A}$ for the normalized $L U$ factorization in Example 4.4.

ConjeCture 4.5. Let $A$ be a nonsingular nearly reducible matrix, and let $P, Q$ be permutation matrices such that $P A Q$ with only nonzero entries on the main diagonal has a normalized $L U$ factorization $P A Q=L U$. Then $L, U \in \mathcal{A}$.

\section{REFERENCES}

[1] T. Britz, D. D. Olesky, and P. van den Driessche. Matrix inversion and digraphs: the one factor case. Electron. J. Linear Algebra, 11:115-131, 2004.

[2] T. Britz, D. D. Olesky, and P. van den Driessche. The Moore-Penrose inverse of matrices with an acyclic bipartite graph. Linear Algebra Appl., 390:47-60, 2004.

[3] R. A. Brualdi and H. Ryser. Combinatorial Matrix Theory. Cambridge University Press, Cambridge, 1991.

[4] D. Carlson, E. Haynsworth, and T. Markham. A generalization of the Schur complement by means of the Moore-Penrose inverse. SIAM J. Appl. Math., 26:169-175, 1974.

[5] F. R. Gantmacher. The Theory of Matrices. Chelsea, New York, 1960.

[6] R. A. Horn and C. R. Johnson. Matrix Analysis. Cambridge University Press, Cambridge, 1985.

[7] C. R. Johnson and J. S. Maybee. Qualitative analysis of Schur complements. DIMACS, Ser. Discrete Math. Theor. Comput. Sci., 4:359-365, 1991.

[8] C. R. Johnson, D. D. Olesky, and P. van den Driessche. Sign determinancy in $L U$ factorization of P-matrices. Linear Algebra Appl., 217:155-166, 1995.

[9] E. Moore. On the reciprocal of the general algebraic matrix. Bull. Amer. Math. Soc., 26:394395, 1920.

[10] D. V. Ouellette. Schur complements and statistics. Linear Algebra Appl., 36:187-295, 1981.

[11] R. Penrose. A generalized inverse for matrices. Proc. Camb. Philos. Soc., 51:406-413, 1955.

[12] G. W. Stewart. Matrix Algorithms, Vol. I: Basic Decompositions. SIAM, Philadelphia, 1998. 